\title{
Design evolution enhances patient compliance for low-intensity pulsed ultrasound device usage
}

\author{
Neill M Pounder \\ John $T$ Jones \\ Kevin J Tanis \\ Bioventus LLC, Durham, NC, USA
}

Video abstract

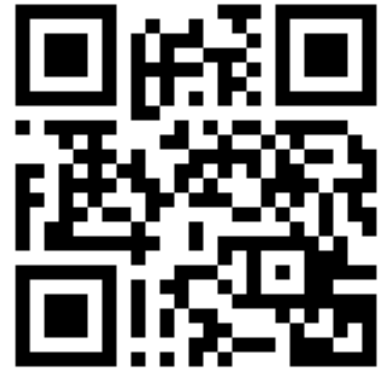

Point your SmartPhone at the code above. If you have a QR code reader the video abstract will appear. Or use: http:://youtu.be/3WMVzrro_04
Correspondence: Neill M Pounder Bioventus LLC, 472I Emperor Boulevard, Suite 100, Durham, NC 27703, USA

Tel + I 9194746738

Email neill.pounder@bioventusglobal.com
This article was published in the following Dove Press journal:

Medical Devices: Evidence and Research

30 November 2016

Number of times this article has been viewed

\begin{abstract}
Poor patient compliance or nonadherence with prescribed treatments can have a significant unfavorable impact on medical costs and clinical outcomes. In the current study, voice-of-the-customer research was conducted to aid in the development of a next-generation low-intensity pulsed ultrasound (LIPUS) bone healing product. An opportunity to improve patient compliance reporting was identified, resulting in the incorporation into the next-generation device of a visual calendar that provides direct feedback to the patient, indicating days for which they successfully completed treatment. Further investigation was done on whether inclusion of the visual calendar improved patient adherence to the prescribed therapy ( 20 minutes of daily treatment) over a 6-month period. Thus, 12,984 data files were analyzed from patients prescribed either the earlier- or the next-generation LIPUS device. Over the 6-month period, overall patient compliance was $83.8 \%$ with the next-generation LIPUS device, compared with $74.2 \%$ for the previous version $(p<0.0001)$. Incorporation of the calendar feature resulted in compliance never decreasing below $76 \%$ over the analysis period, whereas compliance with the earlier-generation product fell to $51 \%$. A literature review on the LIPUS device shows a correlation between clinical effectiveness and compliance rates more than $70 \%$. Incorporation of stakeholder feedback throughout the design and innovation process of a next-generation LIPUS device resulted in a measurable improvement in patient adherence, which may help to optimize clinical outcomes.
\end{abstract}

Keywords: LIPUS, ultrasound, compliance, patient adherence, medical device design

\section{Introduction}

Patient compliance, or nonadherence, to medication and medical treatments has a significant impact on health system costs. Between $3 \%$ and $10 \%$ of total US annual health care costs have been attributed to waste due to lack of compliance, representing $\$ 100-\$ 300$ billion of avoidable expenditure. ${ }^{1}$ A systematic review has found that patient medication compliance across 76 published studies averaged $71 \%{ }^{2}$ Furthermore, DiMatteo et $\mathrm{al}^{3}$ showed that patient adherence and treatment outcome are strongly related. Patient adherence reduced the risk of a poor outcome by $26 \%$, and if a patient was compliant, the odds of a good outcome were almost three times higher than if the patient was not compliant (standardized odds ratio).

The aim of this study was to determine whether patient adherence to low-intensity pulsed ultrasound (LIPUS) therapy, delivered by a prescription US Class III medical device, is improved if the patient is given direct feedback of compliance. Such information could help improve the design of medical devices by providing a rationale to incorporate features to improve patient adherence. 
Exogen ${ }^{\circledR}$ is indicated for the accelerated healing of certain fresh bone fractures ${ }^{4,5}$ and for the treatment of established nonunions $s^{6,7}$ (excluding skull and vertebrae), and it is typically used by the patient in an in-home setting. Treatment consists of one daily 20-minute application of the device over the site of fracture or nonunion; the LIPUS signal is delivered via a gel-coupled transducer. For an earlier-generation LIPUS device (Exogen ${ }^{\circledast} 4000+$; Bioventus LLC, Durham, NC, USA), the date, time, and duration of the treatment was recorded in the device's internal memory; however, no readily visible information on how compliant the patient was to the treatment protocol was provided. Following development of the next-generation device (Exogen ${ }^{\circledast}$ Ultrasound Bone Healing System; Bioventus LLC), which incorporates direct feedback on a patient's daily compliance with their prescribed treatment, it was hypothesized that patient adherence would be improved. The visual compliance calendar enabling direct feedback to the patient was the key change to the next-generation device, thus facilitating comparison of the impact of design changes between devices. In addition, patient compliance with the commercially used products was compared to the compliance rates and outcomes reported for clinical trials.

The development of the next-generation LIPUS device for in-home use started with user insights. The innovation process used to develop the medical device and balance the various stakeholder inputs has previously been described. ${ }^{8}$ The development process consisted of three phases: discovery, concept envisioning, and design refinement.

\section{Discovery phase}

To obtain stakeholder input, ${ }^{8}$ voice-of-the-customer research was conducted with patients, physicians, and payers/insurance companies. The physician prescribes the medical device and periodically reviews progress with the patient. The patient is responsible for performing treatment daily without supervision, while the payer is the organization covering the cost of the treatment. The voice-of-the-customer research consisted of one-on-one interviews in the patient's home or the physician's office, together with panel meetings with payers and physicians. More than 700 separate voice-ofcustomer insights were documented. Physicians identified the need to track compliance and link patient usage to their outcomes. Payers were also interested in patient adherence from a cost-effectiveness standpoint. With earlier-generation units, physicians solely relied on the patient's feedback to determine whether they were using the device appropriately, and as such, patient adherence to the prescription was not rigorously monitored.

\section{Concept envisioning phase}

A range of product concepts was developed and tested through patient and physician interviews. The concepts were deliberately simple, with minor design aesthetics so that patients and physicians would react to the idea, rather than the imagery. Patients valued the idea of the screen, and physicians expressed interest, particularly in monitoring atrisk patients, such as smokers and diabetics. ${ }^{9-11}$

\section{Design refinement phase}

To finalize the inclusion of the compliance calendar, as well as other features, telephone interviews were conducted with 13 medical directors from insurance companies, and a quantitative marketing study with 162 physicians was performed. Thus, $80 \%$ of physicians concurred that addition of a compliance calendar would help their patients to be more adherent to treatment therapy. There was a clear preference for the information to be presented in a calendar format on the device (55\%) rather than numerically $(22 \%)$ or via a website $(23 \%)$. The final selected concept was a compliance calendar incorporated into the face of the device, with the patient attending follow-up appointments with their LIPUS unit, to enable their physician to review compliance in a collaborative manner. The initial calendar concept, together with the final version, is shown in Figure 1.

\section{Final design}

For the next-generation LIPUS device, the calendar screen displays a checkmark if a patient completes a 20-minute treatment correctly (ie, one continuous treatment). The screen shows a double checkmark if a patient completes two 20-minute treatments within a given day, or a double checkmark with a plus symbol for three or more treatments.

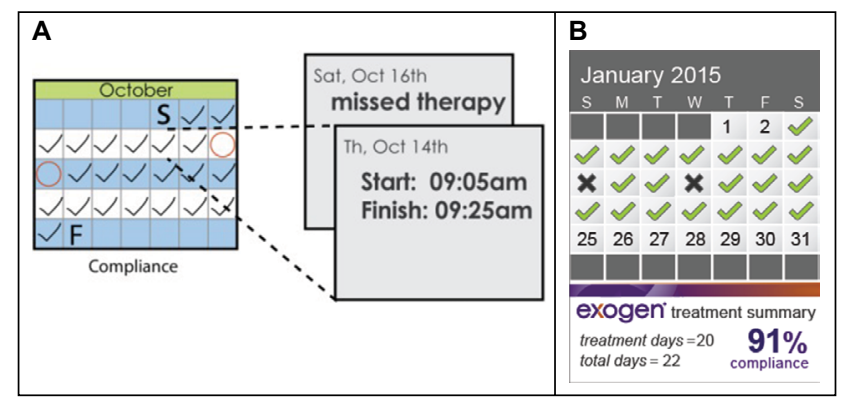

Figure I Refinement of calendar display design.

Note: (A) Initial concept and (B) final compliance calendar for the next-generation LIPUS device.

Abbreviation: LIPUS, low-intensity pulsed ultrasound. 
In each of these cases, the compliance percentage is recorded as the same. The typical prescription is for a single 20-minute treatment, and it is not possible to have more than $100 \%$ compliance. Lastly, a cross mark is displayed if a patient does not complete a 20-minute treatment on a given day. Completing two treatments on a day after a missed treatment does not improve the compliance percentage. The compliance calendar provides simple indicators of patient adherence via checkmarks and crosses, as well as a percentage number. The final updated product was launched in the US market in June 2014.

\section{Materials and methods Data collection}

The need for Institutional Review Board was waived under the Code of Federal Regulations Title 45, part 46.101(b)(4) as it involved the collection and analysis of existing deidentified data. All subjects gave written informed consent prior to receiving their device. Patient usage files from the years 2006 to 2011, as well as from 2014 to 2015, were collected from the earlier-generation and next-generation LIPUS devices, respectively. The earlier-generation unit used a primary battery pack. Once the battery was depleted, and if the patient was continuing with treatment, the device was returned to the manufacturer for the battery to be replaced. During this process, the internal memory data were accessed, and the patient's device usage data were retrieved. The nextgeneration LIPUS device uses a rechargeable battery, and the initial service limit was set to 185 treatments of 20 minutes each. Once the service limit is reached, the unit alerts the user to contact customer service, whereupon the device is returned for servicing and the usage data are similarly downloaded. Customer profiles consist of fresh and nonunion fractures in a range of fracture locations.

The device usage data stored within the LIPUS devices consist of the following: day, date, and time of treatment start; the duration of treatment; and whether any errors occurred during the treatment. A line of data was generated each time the device was switched on. All factory-recorded test activations, either during original calibration or servicing, were removed, and files containing no data or generated as a result of device failures were not included in the assessment. For statistical analyses, $p$-values were obtained from a repeated-measures generalized estimable equation model.

\section{Compliance data analysis}

The LIPUS device is indicated for one 20-minute treatment per day, and patients are considered to be compliant on a given day if they complete one continuous treatment greater than
18 minutes. A patient is not considered to be compliant if he/ she completes numerous partial treatments totaling $20 \mathrm{~min}$ utes, eg, two 10-minute treatments. If a patient completes more than one treatment per day, the level of compliance is not increased. The LIPUS devices assessed in this study were returned at different stages of a patient's overall treatment course. In the case of the earlier-generation LIPUS units, the devices were returned once the primary battery was depleted, which varied between 190 and 230 treatments. In the case of the next-generation device, it is returned when the servicing limit of 185 treatments is reached. To enable a like-for-like comparison, both data sets were compared over the first 185 days. Compliance was determined for each day starting with the first date of a full 18-minute treatment or greater, continuing until the last date of operation or until day 185 , whichever occurred first.

\section{Results and discussion}

For the earlier-generation LIPUS device data set, 10,763 valid patient usage files over the period of 2006-2011 were assessed. A total of 2,221 usable data files for the nextgeneration device were obtained from 2014 to 2015 . Overall, average patient compliance for the earlier-generation LIPUS device was $74.2 \%$, while compliance for the next-generation LIPUS device was significantly higher, at $83.8 \%$, over the 185 -day treatment period $(p<0.0001)$. As shown in Figure 2, daily compliance for the earlier-generation device started at $89.9 \%$ over the first 30 days of use, gradually falling to $51.2 \%$ at the end of the analysis period. In comparison, compliance

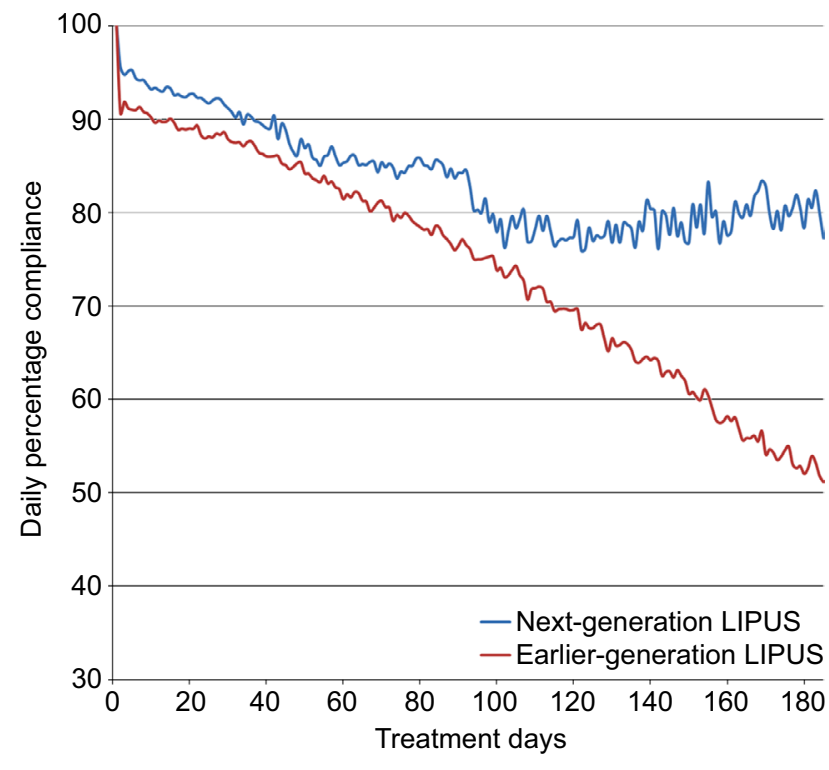

Figure 2 Comparison of patient compliance between earlier-generation and nextgeneration LIPUS devices.

Abbreviation: LIPUS, low-intensity pulsed ultrasound. 
Table I Reported compliance rate in controlled clinical studies of LIPUS treatment

\begin{tabular}{|c|c|c|c|c|c|}
\hline Authors & Compliance, \% & Success? & Number of patients & Study & Bone \\
\hline \multicolumn{6}{|c|}{ High-compliance trials } \\
\hline Kristiansen et al ${ }^{5}$ & 83 & Yes & 61 & RCT & Radius \\
\hline Nolte et $\mathrm{al}^{7}$ & 72 & Yes & 29 & Non-RCT & Mixed \\
\hline Tsumaki et al ${ }^{12}$ & 100 & Yes & 21 & $\mathrm{RCT}$ & Tibia \\
\hline Handolin et al ${ }^{17}$ & 95 & No & 30 & $\mathrm{RCT}$ & Malleolar \\
\hline Gebauer et $\mathrm{al}^{6}$ & 89 & Yes & 67 & Non-RCT & Mixed \\
\hline Rutten et $\mathrm{al}^{13}$ & 98 & Yes & 71 & Non-RCT & Tibia \\
\hline Lubbert et al ${ }^{18}$ & 93 & No & 101 & RCT & Clavicle \\
\hline Schofer et al $^{14}$ & 91 & Yes & 101 & RCT & Tibia \\
\hline Roussignol et al ${ }^{15}$ & 95 & Yes & 59 & Non-RCT & Mixed \\
\hline Matsubara et al ${ }^{16}$ & 79 & Yes & 94 & Non-RCT & Mixed \\
\hline \multicolumn{6}{|c|}{ Low-compliance trials } \\
\hline Emami et a ${ }^{19}$ & 57 & No & 32 & $\mathrm{RCT}$ & Tibia \\
\hline TRUST study ${ }^{20}$ & 43 & No & 501 & $\mathrm{RCT}$ & Tibia \\
\hline
\end{tabular}

Abbreviations: LIPUS, low-intensity pulsed ultrasound; RCT, randomized controlled trials; TRUST, Trial to Re-evaluate Ultrasound in the Treatment of Tibial Fractures.

for the next-generation device was $93.3 \%$ over the first 30 days, falling to $84.2 \%$ at day 90 . Beyond 90 days, the compliance was maintained at approximately $80 \%$, ranging from $76 \%$ to $84 \%$. Compliance for the next-generation LIPUS device did not decrease below $76 \%$ over the 6 -month period. The compliance over the first 3 months is similar between the devices but diverges after approximately 3 months of use when the compliance for the earlier-generation device declines substantially. Incorporation of the visual calendar into the design of the next-generation device has a positive impact on reinforcing compliance, potentially due to more direct patient feedback on device usage.

In order to better understand the correlation between patient treatment adherence and clinical outcomes, information was then compared from controlled clinical studies using the earlier-generation devices where compliance with LIPUS treatment until the fracture was healed was reported (Table 1). In LIPUS trials with a successful outcome, ${ }^{5-7,12-16}$ wherein the study met the protocol-defined end point, the average compliance was $88 \%$ (range: $72 \%-100 \%$ ), whereas for trials where the primary end point was not met, the compliance was 54\% (range: 43\%-95\%). ${ }^{17-20}$ Moreover, eight out of ten trials with patient compliance higher than $72 \%$ reported a successful outcome, ${ }^{5-7,12-16}$ whereas two clinical trials that reported poor patient compliance resulted in unsuccessful outcomes. In the two trials that had high compliance but did not result in a successful outcome, ${ }^{17,18}$ other factors may have confounded the results. In the case of Lubbert et al, ${ }^{18}$ the outcome was measured by the patient's subjective assessment of healing, with no corresponding radiographic data used to judge fracture status. Handolin et $\mathrm{al}^{17}$ acknowledged that their clinical trial was not powered to show statistical significance.
In addition, subjects in that trial were instructed to use the device for 6 weeks, whereas the device standard instructions for use note that treatment should continue until the fracture has healed. By 6 weeks, fracture callus formation was only seen in $27 \%(8 / 30)$ of subjects. ${ }^{17}$

Patient compliance for LIPUS device usage had not previously been analyzed in a real-world clinical setting. The inclusion of direct feedback for patient usage of the LIPUS device, through incorporation of a visible calendar feature, had a significant impact on patient compliance. The incorporation of such feedback into the LIPUS bone healing system, together with its short daily treatment time, supports patient adherence with therapy. In addition, physician-patient communication has been shown to have a positive correlation with patient adherence. ${ }^{21}$ Niikura et $\mathrm{al}^{22}$ showed that an explanatory leaflet, together with a verbal explanation, enabled their patients to comply with the LIPUS therapy at a rate of $96.2 \%$. Roussignol et al ${ }^{15}$ had a similarly high compliance rate of $95 \%$, and the study protocol required that the clinical trial subject bring their device to each follow-up visit such that compliance could be checked.

\section{Conclusion}

Thus, the benefits of improved compliance monitoring with the next-generation LIPUS device, combined with the ability of clinicians to more rigorously monitor device usage, appears to be beneficial in efforts to optimize clinical effectiveness. For the low-compliance clinical trials noted herein, improved physician-patient communication and following the standard instructions for use may have improved patient adherence and thus the clinical outcome. 


\section{Disclosure}

The authors are employees of Bioventus LLC, which manufactures and sells a LIPUS device known as Exogen ${ }^{\circledR}$. The authors report no other conflicts of interest in this work.

\section{References}

1. Iuga AO, McGuire MJ. Adherence and health care costs. Risk Manag Healthc Policy. 2014;7:35-44.

2. Claxton AJ, Cramer J, Pierce C. A systematic review of the associations between dose regimens and medication compliance. Clin Ther. 2001;23(8):1296-1310.

3. DiMatteo R, Giordani PJ, Lepper HS, Croghan TW. Patient adherence and medical treatment outcomes: a meta-analysis. Med Care. 2002;40(9):794-811.

4. Heckman JD, Ryaby JP, McCabe J, Frey JJ, Kilcoyne RF. Acceleration of tibial fracture-healing by non-invasive, low-intensity pulsed ultrasound. J Bone Joint Surg Am. 1994;76A(1):26-34.

5. Kristiansen TK, Ryaby JP, McCabe J, Frey JJ, Roe LR. Accelerated healing of distal radius fractures with the use of specific, low-intensity ultrasound. J Bone Joint Surg Am. 1997;79A(7):961-973.

6. Gebauer D, Mayr E, Orthner E, Ryaby JP. Low-intensity pulsed ultrasound: effects on nonunions. Ultrasound Med Biol. 2005;31(10):1391-1402.

7. Nolte PA, van der Krans A, Patka P, Janssen IMC, Ryaby JP, Albers GHR. Low-intensity pulsed ultrasound in the treatment of nonunions. J Trauma. 2001;51(4):693-703.

8. de Ana FJ, Umstead KA, Phillips GJ, Conner CP. Value driven innovation in medical device design: a process for balancing stakeholder voices. Ann Biomed Eng. 2013;41(9):1811-1821.

9. Bishop JA, Palanca AA, Bellino MJ, Lowenberg DW. Assessment of compromised fracture healing. J Am Acad Orthop Surg. 2012;20(5): 273-282.

10. Hernandez RK, Do TP, Critchlow CW, Dent RE, Jick SS. Patient-related risk factors for fracture-healing complications in the United Kingdom General Practice Research Database. Acta Orthop. 2012;83(6):653-660.

11. Lynch JR, Taitsman LA, Barei DP, Nork SE. Femoral nonunion: risk factors and treatment options. J Am Acad Orthop Surg. 2008;16(2): 88-97.
12. Tsumaki N, Kakiuchi M, Sasaki J, Ochi T, Yoshikawa H. Low-intensity pulsed ultrasound accelerates maturation of callus in patients treated with opening-wedge high tibial osteotomy by hemicallotasis. $J$ Bone Joint Surg Am. 2004;86A(11):2399-2405.

13. Rutten S, Nolte PA, Guit GL, Bouman DE, Albers GHR. Use of low-intensity pulsed ultrasound for posttraumatic nonunions of the tibia: a review of patients treated in The Netherlands. J Trauma. 2007;62(4):902-908.

14. Schofer MD, Block JE, Aigner J, Schmelz A. Improved healing response in delayed unions of the tibia with low-intensity pulsed ultrasound: results of a randomized sham-controlled trial. BMC Musculoskelet Disord. 2010;11:229.

15. Roussignol X, Currey C, Duparc F, Dujardin F. Indications and results for the Exogen ${ }^{\mathrm{TM}}$ ultrasound system in the management of non-union: a 59-case pilot study. Orthop Traumatol Surg Res. 2012;98(2):206-213.

16. Matsubara T, Sano M, Morioka S, et al. Compliance of low-intensity pulsed ultrasound (LIPUS) for fresh fractures. J Orthop Trauma. 2015;29(5 Suppl):S1-S6.

17. Handolin L, Kiljunen V, Arnala I, Pajarinen J, Partio EK, Rokkanen P. The effect of low intensity ultrasound and bioabsorbable self-reinforced poly-L-lactide screw fixation on bone in lateral malleolar fractures. Arch Orthop Trauma Surg. 2005;125(5):317-321.

18. Lubbert PHW, van der Rijt RHH, Hoorntje LE, van der Werken C. Low-intensity pulsed ultrasound (LIPUS) in fresh clavicle fractures: a multi-centre double blind randomised controlled trial. Injury. 2008;39(12):1444-1452.

19. Emami A, Petren-Mallmin M, Larsson S. No effect of low-intensity ultrasound on healing time of intramedullary fixed tibial fractures. J Orthop Trauma. 1999;13(4):252-257.

20. Trial to evaluate ultrasound in the treatment of tibial fractures (TRUST). Available from: https:/clinicaltrials.gov/show/NCT00667849. NLM identifier: NCT00667849. Published November 4, 2015. Accessed June 21, 2016.

21. Haskard Zolnierek KB, DiMatteo MR. Physician communication and patient adherence to treatment: a meta-analysis. Med Care. 2009;47(8):826-834.

22. Niikura T, Lee SY, Iwakura T, Kurosaka M. Improving patient compliance with low-intensity pulsed ultrasound (LIPUS) treatment. J Orthop Trauma. 2014;28(6 Suppl):S1-S6.
Medical Devices: Evidence and Research

\section{Publish your work in this journal}

Medical Devices: Evidence and Research is an international, peerreviewed, open access journal that focuses on the evidence, technology, research, and expert opinion supporting the use and application of medical devices in the diagnosis, monitoring, treatment and management of clinical conditions and physiological processes. The identification of novel

\section{Dovepress}

devices and optimal use of existing devices which will lead to improved clinical outcomes and more effective patient management and safety is a key feature. The manuscript management system is completely online and includes a quick and fair peer-review system. Visit http://www. dovepress.com/testimonials.php to read real quotes from authors. 